This is a postprint version of the following published document:

Contreras, Luis M., et al. Orchestration of Crosshaul slices from federated administrative domains. EuCNC 2016, "The dawn of 5G", Papers (of the) European Conference on Networks and Communications, 27-30 June 2016, Athens, Greece. IEEE, 2016 (5 pp.)

DOI: https://doi.org/10.1109/EuCNC.2016.7561036

C 2016 by IEEE. Personal use of this material is permitted. Permission from IEEE must be obtained for all other uses, in any current or future media, including reprinting/republishing this material for advertising or promotional purposes, creating new collective works, for resale or redistribution to servers or lists, or reuse of any copyrighted component of this work in other works. 


\section{Orchestration of Crosshaul Slices From Federated Administrative Domains}

\author{
Luis M. Contreras \\ Telefónica I+D / Telefónica Global CTO Unit \\ Madrid, Spain \\ luismiguel.contrerasmurillo@telefonica.com \\ Carlos J. Bernardos, Antonio de la Oliva \\ Universidad Carlos III de Madrid \\ Leganés, Spain \\ \{cjbc, aoliva\}@it.uc3m.es
}

\author{
Xavier Costa-Pérez \\ NEC Laboratories Europe \\ Heidelberg, Germany \\ xavier.costa@neclab.eu \\ Riccardo Guerzoni \\ Huawei Technologies Düsseldorf GmbH \\ Munich, Germany \\ riccardo.guerzoni@huawei.com
}

\begin{abstract}
With the advent of 5G networks, more dynamicity and flexibility will be needed for the deployment of services with very distinct requirements. Crosshaul areas (those integrating fronthaul and backhaul) are especially critical because of the variability of the demand and the cost of the (own) network deployment, which in many cases is jeopardized by the huge level of investment needed. A common market place to trade the required networking and computing facilities (in the form of a slice) in a multi-domain federated environment is envisaged as the solution for easing the adaptation to future demands. This paper proposes to develop the concept of multi-domain Crosshaul by enabling the dynamic request of Crosshaul slices through a multi-provider exchange.
\end{abstract}

Keywords—multidomain; fronthaul; backhaul; SDN; NFV; exchange; 5GEx; 5 G-Crosshaul.

\section{INTRODUCTION}

The progressing immersion into digital economy, the fierce competition from Over-The-Top (OTT) service providers, the dynamicity introduced by hosting virtualized service functions into the cloud, and the demand of permanent connectivity to digital services are changing the way communication services are provided. 5G [1] is the next step on this change, proposing a pervasive availability of access capacity even in ultra-dense population areas. Shorter latency, fast Time-To-Market (TTM) for any kind of service, and a better and consistent quality of experience (independent of the point of attachment to the network) are key aspects to address.

Software Defined Networking (SDN) and Network Functions Virtualization (NFV) will be two enablers to achieve that. Basically, network programmability will facilitate rapid provisioning of connectivity, and the decoupling of functions from dedicated network elements will provide the means to dynamically adapt to a changing demand.

However, those enablers together could not be enough to address future scenarios from a service provider perspective. The deployment of network infrastructure is a time consuming process, requiring careful business planning to support the necessary investments, in order to be ready for service delivery at the proper time when the demand arises. In addition to that, infrastructure ownership may be unsustainable in a revenuedecreasing scenario, driving to infrastructure sharing to reduce the total cost associated to the service provisioning.

In this situation, the idea of leasing virtualized networking and computing environments is gaining momentum. Thus, Infrastructure Providers (InP) can play the role of facilitators for service providers in order to lower the Total Cost of Ownership (TCO) [2], simplify the network architecture, and streamline the operation and their associated costs.

This can be significantly the case for access and aggregation networks. Uncertainty in the number of end users, their distribution and mobility patterns and heterogeneous service requirements (from data intensive residential-like service to flow-intensive machine-to-machine connections) make unpredictable and dynamic the demand of connectivity and network services.

Specifically, for the aggregation stages, close to the radio access (what is typically known as a conjunction of fronthaul and backhaul areas, or Crosshaul in the context of this paper) seems quite appealing to introduce flexibility to dynamically adapt the deployed resources to the concrete demand. The demand of dynamic resource allocation involve networking but also computing facilities, in order to flexibly deploy services and host content at the edge, thus saving core network capacity and decreasing service latency.

Furthermore, the capability of combining resources from different InPs can provide further flexibility and adaptation to diverse end user behaviors and performance requirements, thus overcoming current limitations imposed by tight coupling of service and infrastructure.

Then two possible multi-domain cases can be taken into consideration: (i) composition of administratively separated Crosshaul domains; and (ii) composition of end-to-end administratively separated domains (including Core Network, Crosshaul and Radio Access Network -RAN-). This paper focuses on the first case. 
There is yet a gap to reach the goal of hosting Crosshaul in a multi-domain federated infrastructure: a market place where networking and computing facilities are traded. An extension of the traditional concept of telco exchange is needed, covering new needs and capabilities, such as offering resource slices for deployment of the services requested by third party service providers.

This paper proposes to develop the concept of multidomain Crosshaul by presenting an architectural framework enabling the dynamic request of Crosshaul slices through a multi-provider exchange. Section II introduces a view on $5 \mathrm{G}$ services and requirements to be supported on these dynamically bound (virtualized) infrastructures. Section III presents in detail the ideas behind the Crosshaul areas which are the focus of the analysis. Section IV defines the concept of exchange in the context of $5 \mathrm{G}$, with a wider scope with respect to the conventional approach. Section $\mathrm{V}$ describes the composition of multi-domain Crosshaul areas. Finally, Section VI proposes future work areas and presents some conclusions.

\section{5G SERVICES AND REQUIREMENTS}

Although there is no clear consensus yet on how 5G networks will look like, it is clear that they will be characterized by supporting new extremely demanding use cases, compared to what we have now. Among these use cases, there are several that are often mentioned: $i)$ provision of extremely high throughput to users (e.g., to support $4 \mathrm{~K}$ video streaming); ii) provide simultaneous connectivity to a very large number of low cost, low capability and low performance devices (supporting the Internet-of-Things era); iii) deploy ultra-dense access networks imposing high mobility rates; and iv) guarantee extremely low latency requirements to support the upcoming tactile Internet applications. These scenarios pose quite challenging requirements on all segments of the network, but especially on the radio access and transport [3].

If we focus for a while on the transport aspects of $5 \mathrm{G}$ networks, it is clear that networks will have to evolve for supporting massive traffic volumes, requiring as well to be capable of providing high capacity on demand to react to varying demand conditions. This will require different transport network technologies (e.g., optical fiber, microwave, etc.) to be able to efficiently inter-operate by means of a common control plane. Additionally, fast re-configurability will also be needed in order to dynamically react to load demands.

Moving now to the service aspects of future 5G networks, there is the trend of provisioning services as close as possible to where users are, as one (more) mechanism to reduce CapEx. The effort being done at the ETSI Mobile Edge Computing (MEC) ISG is a very good example of this trend, looking into the opportunity of dynamically instantiating services close to the edge, re-using resources initially deployed just for pure connectivity purposes.

Software Defined Networking (SDN) and Network Function Virtualization (NFV) techniques are commonly identified as solutions to the aforementioned challenges [4].

\section{CROSSHAUL AREAS}

The $5^{\text {th }}$ Generation of communication networks will not only focus on providing more bandwidth to the final user through a novel air interface, but will also work on redesigning the overall network infrastructure, including access, transport and core network segments, to achieve a network with a lower cost of deployment and operation while improving the available services to the end-users.

Two key characteristics define this new generation of transport networks, which will integrate the fronthaul and backhaul segments in an integrated network, the Crosshaul. On the one hand, the concept of multi-tenancy, where a network is shared among different customers or tenants, will be an integral part of the design of future networks, designed from scratch to be shareable, reducing their CapEx and OpEx costs and increasing their usage efficiency. On the other hand, networks will be tailor made for the needs of each tenant. The customization of network services and characteristics will allow different network views to coexist on top of a common infrastructure.

In the remaining of this section we present the overall architecture of the $5 \mathrm{G}$-Crosshaul project ${ }^{1}$, which takes as basis the two characteristics explained above to create the next generation integrated transport network.

\section{A. 5G-Crosshaul}

5G-Crosshaul [5] aims at developing an adaptive, shareable, cost-efficient 5G transport network solution integrating the fronthaul and backhaul segments of the network [6], [7]. This transport network will flexibly interconnect distributed 5G radio access systems and core network functions, hosted on in-network cloud nodes. The implementation of three novel building blocks is considered in 5G-Crosshaul: $(i)$ a control infrastructure using a unified, abstract network model for control plane integration (Crosshaul Control Infrastructure, XCI); (ii) a unified data plane encompassing innovative high-capacity transmission technologies and novel latency-deterministic switch architectures (Crosshaul Forwarding Element, XFE); and (iii) a set of computing capabilities distributed across the network (Crosshaul Processing Units, XPUs).

Crosshaul will greatly simplify network operations despite growing technological diversity. It will hence enable system-wide optimization of Quality of Service (QoS) and energy usage as well as network-aware application development.

Crosshaul follows a unique approach towards the integration of the different network segments (fronthaul and backhaul) into a common transport stratum. In order to integrate the different nature of the fronthaul and backhaul traffic (with their very disparate requirements) and the different technologies that can be used to transport them, a new common transport framing format is defined (the XCF, Crosshaul Common Frame) which is used to perform the forwarding within the Crosshaul. This XCF is based on MAC-

${ }^{1}$ http://5g-crosshaul.eu/ 
in-MAC [8] Ethernet, and all traffic going into a Crosshaul area is adapted to this frame format. In this way, Crosshaul can leverage all the work performed in IEEE 802.1 (IEEE 802.1TSN and IEEE802.1CM) to transport flows with stringent delay requirements in Ethernet-based networks.

In order to be able to control a network with characteristics like the ones of Crosshaul (transporting traffic with very extreme properties), it is needed a very flexible and adaptable control plane, able to orchestrate all the network, storage and computational resources. Figure 1 presents the 5G-Crosshaul $\mathrm{XCI}$ architecture showcasing a multi-tenant scenario.

The transport network envisioned by 5G-Crosshaul will serve as a transport stratum to three kind of tenants:

- A Network Operator (NO), owner of the physical infrastructure and in charge of orchestrating the physical resources among the different tenants of the network. It is depicted as VIP (Virtual Infrastructure Provider) at the bottom of Figure 1.

- A Mobile Virtual Network Operator (MVNO), which uses a set of virtualized resources from the Crosshaul to interconnect its infrastructure. In this case, the MVNO will request from the Crosshaul Network Operator a complete virtual infrastructure, managing it and reselling connectivity to other service providers, such as OTT providers. This is the case of Tenant \#1 in Figure 1.

- An Over The Top (OTT) provider, which will make use of the transport infrastructure to connect distributed service points (e.g., a company connecting its own datacenters). This tenant, Tenant\#2 in Figure 1, requires a high level abstraction of the network to operate, and it does not need to resell the virtual infrastructure to upper layers.

As shown in Figure 1, the XCI is compliant with the ETSI NFV architecture [9] for what concern management and orchestration, and complements it by a set of controllers to coordinate networking, storage and computing resources of the Crosshaul. In addition, a new feature, called the Multi-Tenancy Application (MTA), is also being proposed to support infrastructure management in multi-tenancy scenarios.

Although the presented architecture enables an InP to share its network and its associated costs, one point still under study in 5G-Crosshaul is how to build up an end to end network composed of several virtual networks belonging to different Crosshaul domains. This use case is known as the MultiMANO case and one of the approaches that can be used to solve it is to use the mechanisms for multi-domain service composition provided by the $5 \mathrm{G}$ Exchange project ${ }^{2}$, as detailed in the following sections.

\section{B. Enablement of dynamic network service deployments}

5G-Crosshaul will make available slices of compound resources to different tenants for deploying services as composition of virtualized network functions. In addition to that, networking capabilities will be provided accordingly to connect the network functions among them, and to provide connectivity towards the Crosshaul border. A similar approach

\footnotetext{
${ }^{2}$ http://www.5gex.eu/
}

is described in [10], where the authors propose a dynamic virtualized environment for deploying services in telecom networks relying on own infrastructure, even with virtualization capabilities. The concepts of Service Graph (SG) and Forwarding Graph (FG) are introduced, separating service and resource problems at the time of service provision. However multi-domain scenarios are not considered, averting the problem of deploying services on slices leased from different InPs.

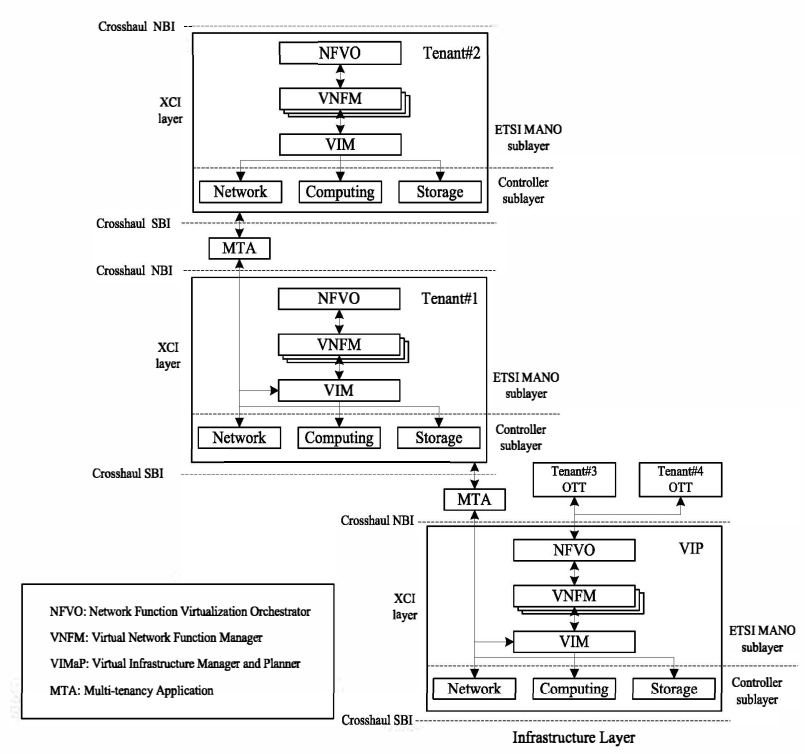

Figure 1:5G-Crosshaul XCI recursive architecture

Management and control of resources and services in multidomain scenarios is a fundamental challenge in 5G networks, especially for Crosshaul applicationss. Network sharing approaches [11], [12] are becoming more and more common because of the potential TCO reduction, then it is required to address this multi-domain environment in the context of SDN and NFV.

\section{5G-EXCHANGE AS MARKET PLACE FOR MULTI-DOMAIN 5G SERVICES}

Currently deployed solutions to steer and manage traffic will not be capable of supporting future 5G traffic. They lack the required flexibility and agility, leading to complex and rigid network policies, which are even worse if multiple domains are involved. Mechanisms such as SDX [13] aim at tackling these issues, but they are not sufficient for the purposes of the scenarios targeted in this paper. What is needed is a framework allowing relevant stakeholders to trade resources and service functions in order to flexibly deploy end-to-end services by involving the required providers. In particular, the need of enabling different 5G-Crosshaul providers to build services encompassing multiple technology and administrative domains. Here is where the concept of $5 \mathrm{G}$ Exchange enters into the picture.

5G Exchange (5GEx) project is defining appropriate mechanisms for supporting multi-domain trading of resources and functions as space for bootstrapping collaboration and 
service delivery between telecommunications operators regarding 5G infrastructure services. Such services and associated resources will play a crucial role in making 5G happen as they provide the foundation of all cloud and networking services apart from the radio interface itself. 5GEx is seen as a facilitator to enable operators to buy, sell, and integrate infrastructure services, enabling one-stop shopping for their customers. It will provide the ability to automatically trade resources, verify requested services, and it will lead to clear billing and charging.

5GEx is building a logical exchange or factory for globally reachable automated $5 \mathrm{G}$ services creation. For the sake of clarity: the exchange is implemented by APIs, not by statically (directly) connected physical appliances. The exchange will allow the resources such as access, connectivity, computing and storage in one network to support different verticals and applications such as e-Health, robotic communications, media, etc. Resources can be traded among federated providers using this exchange, thus enabling service provisioning on a globally reachable basis.

The 5G Exchange scope includes an automated service orchestration, as well as the management and trading of network, storage and cloud resources. The development of a novel technology framework is based on the architectural concepts hereby described.

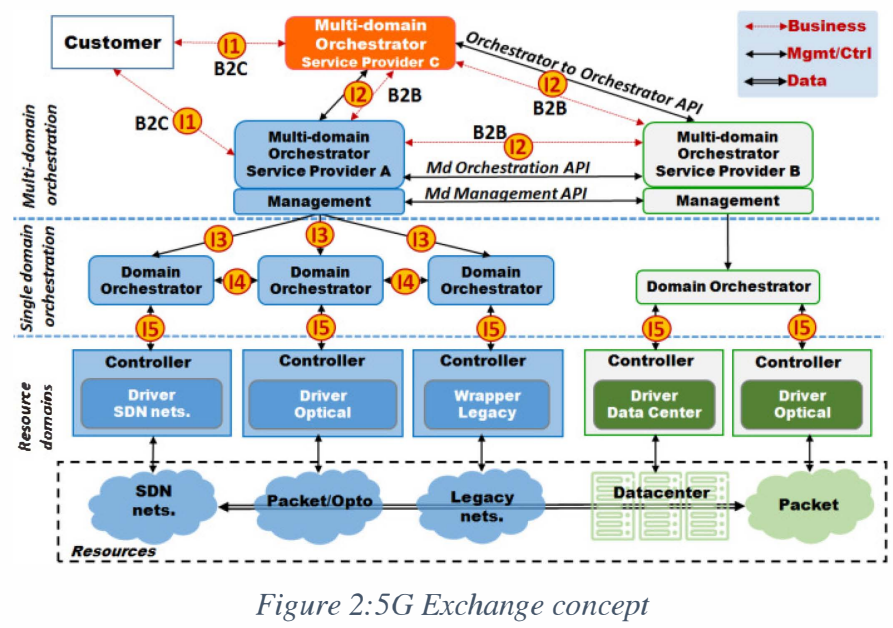

Figure 2 highlights the scope of 5GEx by presenting a logical interworking architecture, showing not only functional entities but also the different APIs between them. The core of 5GEx system is composed of (i) the Multi-domain Orchestrator/Manager, (ii) various domain orchestrators and (iii) collaboration with domain orchestrators and controllers that are in charge of enforcing the requested services on the underlying network, compute, and storage components.

Co-operation between operators takes place at the higher level through the inter-operator orchestration API (I2) that exchanges information, functions and control. This interface also serves for the Business-to-Business relation between operators in complement to the Business-to-Customer API (I1), through which customers request service deployment. The Multi-Domain Orchestrator $(\mathrm{MdO})$ maps service requests into own resource domains and/or dispatches them to other operators through interface (I2). This interaction is performed at $\mathrm{MdO}$ level: each operator $\mathrm{MdO}$ can expose to other operators' MdOs an abstract view of its resource domains and available service functions. Using such an inter-working architecture for multi-domain orchestration will make it possible use cases that are nowadays hard to tackle due to the interactions of multiple heterogeneous actors and technologies.

The MdO enforces the decision through interface (I3) as exposed by Domain Orchestrators, each one orchestrating and managing resource domains through the northbound interfaces (I5) exposed by technology-specific controllers.

The Multi-domain orchestrator in 5GEx is considered to have three main components: $(i)$ the Runtime Engine, which monitors, configures, and runs connectivity and cloud resources across administrative domains; (ii) the Exchange of Functions, which monitors, configures and manages service components across administrative domains; and (iii) the Exchange of Information \& Control, which deploys and runs autonomic management functions.

\section{MULTI-DOMAIN COMPOSITION OF 5G-CROSSHAUL INFRASTRUCTURES}

The 5GEx multi-domain orchestration framework can be used to realize scenarios involving multiple Crosshaul domains belonging to different network operators. 5G-Crosshaul XCIs can play the role of single-domain orchestrators coordinated by 5GEx multi-domain orchestrators. XCI orchestrates both networking resources and compute plus storage within a single administrative domain. Those resources can be offered as dedicated slices in the multi-domain environment. Resource slicing is enabled by the 5G-Crosshaul Multi-Tenancy Application (MTA), which acts as a mediation layer between tenants and the shared infrastructure. In a recursive way, the tenant can program the underlying network facilities and instantiate network functions on the processing units of Crosshaul by using an XCI instance (then stacking XCI control elements) logically isolated from other tenant's XCIs.

Multi-domain Orchestration capabilities are partially supported by the MTA. However, either additional features in the MTA or in a fully new branded application are required in 5G-Crosshaul to fully support inter-operator orchestration and management, i.e. full 5GEx interface (I2) support.

These additional features need to support a number of functionalities for service provision in multi-domain environments, like:

- SLA negotiation, in order to ensure a proper service delivery on the offered Crosshaul slice.

- Service mapping mechanisms in order to assign proper sliced resources to the service request. In the case of 5GCrosshaul this applies to networking (e.g., bandwidth, latency, etc) and compute plus storage (e.g., in terms of CPUs, memory size, type of drive, etc).

- Reporting of Crosshaul metrics, including both the compute and networking substrates, since there is a dependency of the networks function deployed in Crosshaul with regards the hosting facilities and the networking reachability. 
- Proper control and management interfaces to dictate actions in the offered Crosshaul slice, e.g. traffic steering, type of forwarding (packet vs circuit) or network function scale up or down.

The modular nature of the 5G-Crosshaul system architecture permits the introduction of these new functionalities e.g. in the form of a new application for supporting multi-domain environments just implementing interface (I2) of 5GEx, or even as an add-on to MTA.

Figure 3 shows all of these new functionalities represented as a single box embedding also MTA. This could be an implementation option, where a tight binding among MTA and the entity in charge of terminating 5GEx interface (I2) in Crosshaul are part of the same component. Other alternatives could be also possible, and this is a matter of further analysis.

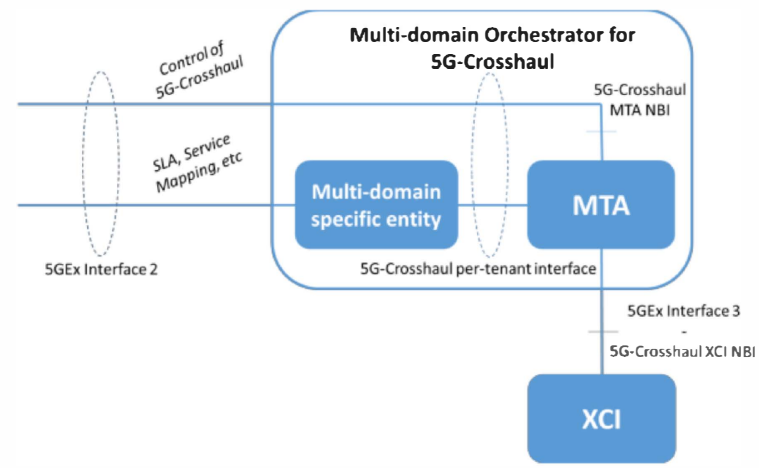

Figure 3:Multi-domain entity for 5G-Crosshaul

\section{CONCLUSIONS AND FUTURE WORK}

The advent of $5 \mathrm{G}$ will accelerate the need of rapid adaptation of the networks to satisfy a changing demand. Such adaptation will leverage on the transformation driven by SDN and NFV, specifically on the new possibilities of providing resource slices on demand from different infrastructure providers. In this context it is essential to define proper ways of composing multi-domain environments in a dynamic form. The concept of exchange needs to evolve towards the idea of not only interconnecting networks for transiting traffic but also to interconnect a variety of resources, in the logical form of slices. In this way, the resources will appear as dedicated for the interconnection purposes, while in reality they are part of a larger infrastructure. This is achieved by means of virtualization and trading of those resources.

The need for this dynamic creation, operation and control of resources will be even exacerbated in the access and aggregation areas, where variability of the demand and cost of (own) deployment cannot justify in some cases the huge level of investment needed. This is the case of Crosshaul areas, giving network continuity between the RAN and the Core Network, and even more, including compute processing elements because of efficiency (i.e., minimizing the need of transiting traffic).

This paper presents an approach to multi-domain Crosshaul composition, combining the architectural frameworks of 5GEx and 5G-Crosshaul projects, and identifying some gaps on existing system architecture definition. To solve this gap a new application (or application component) is proposed to 5GCrosshaul, complementing the architecture with a number of features not yet addressed. With a scope in multi-domain service provision and operation features like SLA management between administrative domains, service mapping, associate metrics, and proper exposition of control and management interfaces are the primary information needed in 5G-Crosshaul for participation in 5GEx as market place for trading of Crosshaul areas.

Future work will address in detail the required information on such interface, with emphasis on Crosshaul, and the relationship with existing MTA application. A future integration between both architectures is foreseen under the bigger umbrella of $5 \mathrm{G}$ architecture.

\section{ACKNOWLEDGMENT}

This work has been supported by the European Community through the projects 5GEx (grant no. 671636) and 5GCrosshaul (grant no. 671598) within the H2020 programme.

\section{REFERENCES}

[1] NGMN Alliance, "5G White Paper", February 2015.

[2] A. Khan, W. Kellerer, K. Kozu, M. Yabusaki, "Network sharing in the next mobile network: TCO reduction, management flexibility, and operational independence", IEEE Communications Magazine, Vol. 49, Num. 10, pp. 134-142, October 2011.

[3] M. Fiorani, P. Monti, B. Skubic, J. Martensson, L. Valcarenghi, P. Castoldi, L. Wosinka, "Challenges for 5G Transport Networks", IEEE ANTS 2014.

[4] C.J. Bernardos, A. de la Oliva, P. Serrano, A. Banchs, L.M. Contreras, H. Jin, J.C. Zúñiga, "An Architecture for Software Defined Wireless Networking", in IEEE Wireless Communication Magazine, Vol. 21, No. 3 , pp. 52-61, 2014

[5] A. De La Oliva, X. Costa Perez, A. Azcorra, A. Di Giglio, F. Cavaliere, D. Tiegelbekkers, J. Lessmann, T. Haustein, A. Mourad, P. Iovanna, "Xhaul: toward an integrated fronthaul/backhaul architecture in 5G networks", IEEE Wireless Communication Magazine, July 2015.

[6] T. Pfeiffer, "Next Generation Mobile Fronthaul and Midhaul Architectures", J. Opt. Commun. Netw., Vol. 7, No. 11, November 2015.

[7] J. Bartelt, P. Rost, D. Wübben, J. Lessmann, B. Melis, G. Fettweis, "Fronthaul and Backhaul Requirements of Flexibly Centralized Radio Access Networks", IEEE Wireless Communication Magazine, October 2015 .

[8] 802.1 ah-2008 - IEEE Standard for Local and metropolitan area networks -- Virtual Bridged Local Area Networks Amendment 7: Provider Backbone Bridges.

[9] ETSI NFV, “Architectural Framework”, ETSI GS NFV 002, V1.1.1, October 2013.

[10] I. Cerrato, A. Palesandro, F. Risso, M. Suñé, V. Vercellone, H. Woesner, "Toward Dynamic Virtualized Network Services in Telecom Operator Networks", Elsevier Computer Communications, in press, 2015.

[11] X. Costa-Perez, J. Swetina, G. Tao, R. Mahindra, S. Rangarajan, "Radio access network virtualization for future mobile carrier networks", IEEE Comm. Mag., Vol. 51, No. 7, July 2013.

[12] T.K. Forde, I. Macaluso, L.E. Doyle, "Exclusive sharing and virtualziation of the cellular network", in Proc. IEEE Symp. On New Frontiers in Dynamic Spectrum Access Networks (DysPAN), May 2011.

[13] A. Gupta, L. Vanbever, M. Shahbaz, S. Donovan, B. Schlinker, N. Feamster, J. Rexford, S. Shenker, R. Clark, E. Katz-Bassett, "SDX: A Software Defined Internet Exchange", ACM SIGCOMM, Chicago, August 2014. 